\title{
25 Jahre europäische Integration im Rückblick
}

\author{
Jacques Santer*
}

\section{Stationen europäischer Integration}

Ich sehe meinen Beitrag zur Würdigung von 25 Jahren ,Jahrbuch der europäischen Integration“ in einer persönlichen Rückschau aus dem Blickwinkel des Politikers, der in seiner aktiven politischen Laufbahn während der letzten 25 Jahre alle europäischen Institutionen durchlaufen hat und beim Durchmarsch durch die Institutionen auch deren Anpassungen und Umstrukturierungen miterlebt und zum Teil mitgestaltet hat: als junger Parlamentarier war ich von 1974 bis 1979 mit Doppelmandat Mitglied des nicht direkt gewählten Europäischen Parlaments, das damals mit 169 Mitgliedern klein und bescheiden in seinen Befugnissen war, die hauptsächlich konsultativer Natur waren. Nach 1979 wurde ich stets als Spitzenkandidat meiner Partei ins direkt gewählte Europäische Parlament gewählt, konnte mein Mandat aber nicht annehmen, da ich Mitglied einer Regierung war. Zunächst war ich Finanz- und Sozialminister bis 1984 und hatte einen Sitz in den Europäischen Ministerräten in der turbulenten Periode, in der Abwertungen und Währungsanpassungen die Tagesordnung waren. Das Schlagwort von der ,Eurosklerose' machte die Runde. Ab 1984 wurde ich als Ministerpräsident meines Landes Mitglied des Europäischen Rates und übernahm zweimal den Vorsitz: 1985, als wir die Einheitliche Europäische Akte verabschiedeten, und 1991, als wir den Vorsitz der beiden Regierungskonferenzen über die politische Union und die Wirtschafts- und Währungsunion übernahmen, die zum Maastrichter Vertrag führten.

Schließlich wurde ich dann aufgrund des Vetos von John Major gegen die Kandidatur meines Beneluxkollegen Jean-Luc Dehaene, den ich selbst unterstützt hatte, ohne jemals Kandidat gewesen zu sein am 15. Juli 1994 zum Präsidenten der Europäischen Kommission gewählt. So kann sich das Schicksal über Nacht wenden. Ich hatte damals zum dritten Mal die luxemburgischen Landeswahlen gewonnen und nach mühseligen Verhandlungen eine Neuauflage meiner großen Koalition erreicht. Am 13. Juli wurde ich mit meiner neuen Mannschaft vom Großherzog Luxemburgs vereidigt und am 15. Juli nach Brüssel beordert. Einmal mehr hat sich der Mythos der Nasenlänge von Kleopatra bewahrheitet! Nach der Demission meiner Kommission wurde ich als Mitglied des Europäischen Parlaments wiedergewählt, des Parlaments also, das die Demission meiner Kommission beantragt hatte. Ein Parlament allerdings, das inzwischen auf 632 Mitglieder aus 15 Mitgliedstaaten angewachsen war und das seine Selbstbehauptung gegenüber Kommission und Ministerrat in der Ausschöpfung der ihm zustehenden rechtlichen und politischen Mittel durchzusetzen versucht. Nicht zu unterschätzen bleibt in diesem Zusammenhang die Profilierung der europäischen Parteien. Ich selbst war von 1987 bis 1990 zu einem Zeitpunkt Vorsitzender der Europäischen Volkspartei (EVP), als die Christdemokraten versuchten, eine gemeinsame Haltung gegenüber der Wiedervereinigung Deutschlands zu erzielen, was nicht so einfach war.

* Dr. Jacques Santer, Kommissionspräsident a.D., ehemaliger Premierminister Luxemburgs, ehemaliges Mitglied des Europäischen Parlaments und des Europäischen Verfassungskonvents. Vorsitzender des Verwaltungsrates von CLT-UFA, Luxemburg.

Der Text basiert auf dem (gekürzten) Festvortrag von Dr. Jacques Santer anlässlich der Veranstaltung ,25 Jahre Jahrbuch der Europäischen Integration - Rückblick und Ausblick“ des Instituts für Europäische Politik in Zusammenarbeit mit der Nomos Verlagsgesellschaft am 21. September in Berlin. Der Vortragsstil wurde weitgehend beibehalten. 


\section{Die europäische Integration als Erfolgsgeschichte}

Wenn diese Beschreibung meines politischen Lebensweges etwas weitschweifig erscheinen mag, so wollte ich über diese Stationen bereits die entscheidenden Etappen des holperigen und steinigen Weges der europäischen Integration aufzeichnen. Zu Recht schreibt Wilfried Loth in seinem Bilanzartikel für das Jahrbuch der Europäischen Integration 2005, dass ,,in den letzten 25 Jahren seit dem erstmaligen Erscheinen des Jahrbuchs der Europäischen Integration das gemeinschaftliche Europa seine Gestalt beträchtlich verändert hat." Ist diese Europäische Union, wenn wir sie objektiv betrachten und mit Zahlenmaterial konfrontieren, nicht zu einer Erfolgsgeschichte geworden, so erfolgreich, dass Samuel Huntington in einem 1988 publizierten Aufsatz folgendes schreiben konnte: „Sollte die Europäische Gemeinschaft sich politisch zusammenschließen, hätte sie die Bevölkerung, die Ressourcen, den ökonomischen Wohlstand, die Technologie und die potentielle militärische Stärke, die herausragende Macht des 21. Jahrhunderts zu werden. [...] Man kann sich eine europäische, ideologische Ausstrahlung vorstellen, welche der amerikanischen vergleichbar wäre. In der ganzen Welt stehen Menschen vor den Türen amerikanischer Konsulate Schlange, die um Einwanderungsvisa nachsuchen. In Brüssel stehen ganze Länder Schlange, die der Europäischen Gemeinschaft beitreten wollen. Eine Union von demokratischen wohlhabenden, sozial unterschiedlichen Gesellschaften mit gemischten Wirtschaften wäre eine mächtige Kraft auf der Weltbühne. Wenn das nächste Jahrhundert kein amerikanisches mehr sein sollte, dann wahrscheinlich ein europäisches.“

Wie lässt sich die Zuversicht dieses angesehenen amerikanischen Wissenschaftlers erklären? Sein Werk entstand in der Zeit des Aufbruchs der Europäischen Gemeinschaft nach der Relance der 80er Jahre, der Zeit der sogenannten ,Eurosklerose', in der Europa nach den Wehen der Weltwirtschaftskrise Mitte der 1970er Jahre zu gewaltigen Industrieumstrukturierungen unter anderem im Stahl- und Kohlebereich gezwungen wurde. In dieser Zeit, die zur ersten großen Welle von Arbeitslosigkeit geführt hat, kamen selbst noch so gut gemeinte Vorschläge zur Weiterentwicklung der Gemeinschaft wie die von Leo Tindemans, HansDietrich Genscher oder von Kommissionspräsident Gaston Thorn nicht zum Tragen. Erst die Neugestaltung des deutsch-französischen Tandems mit François Mitterrand und Helmut Kohl im Zusammenwirken mit Jacques Delors hat den Integrationsmotor wieder neu angekurbelt.

\section{Der Weg zur Einheitlichen Europäischen Akte}

Kernpunkt der von Delors initiierten Reform war die Verwirklichung des Binnenmarktes mit der Ankündigung eines präzisen Zeitrahmens, was auch ein Meisterstück meiner ersten europäischen Präsidentschaft im zweiten Halbjahr 1985 war. Vorbereitet vom britischen Kommissar Lord Cowfield - was wesentlich war für die Zustimmung von Margaret Thatcher - wurde das sogenannte Binnenmarkt-Weißbuch Gegenstand der Erörterungen des Europäischen Rates in Mailand Ende Juni 1985. Gegen alle Gepflogenheiten und zum größten Erstaunen der Teilnehmer ließ Präsident Bettino Craxi über die Einberufung einer Regierungskonferenz zur Revision der Gemeinschaftsverträge abstimmen. Es war das erste Mal, dass eine solche Prozedur im Europäischen Rat zur Anwendung kam. Obwohl drei Regierungen - Großbritannien, Dänemark und Griechenland - aus unterschiedlichen Gründen dagegen stimmten, bekam die nachfolgende luxemburgische Präsidentschaft den Auftrag, die Regierungskonferenz vorzubereiten.

Wir machten uns an die Arbeit, und in Rekordzeit bereiteten wir eine Vorlage für den Gipfel in Luxemburg für den 3. Dezember vor. Zuerst mussten wir natürlich die Einwände 
der drei skeptischen Regierungen beheben, anschließend musste ein Vertragsvorschlag erarbeitet werden, der zustimmungsfähig war. Das verlangte von einer kleinen Verwaltung ungeheure Anstrengungen: zweimal machte ich selbst die Runde der Partner in den Hauptstädten. Für Großbritannien konnte ich auf die konstruktive Mitarbeit von Geoffrey Howe und Philip Williamson, den Privatsekretär von Margaret Thatcher und späteren Generalsekretär der Kommission, zählen. Dennoch blieben einige strittige Punkte, unter anderem die Bezugnahme auf die Währungsunion, so dass es gegen Mitternacht auf dem Gipfel fast zum Scheitern gekommen wäre, wenn ich nicht eine Unterbrechung der Sitzung angeordnet hätte. So stark hatten sich die Fronten verhärtet. In einem letzten Anlauf erarbeiteten wir eine Paketlösung für die anstehenden Probleme, die schlussendlich die allgemeine Zustimmung fand.

Diese Einheitliche Europäische Akte, die am 3. Dezember 1985 beschlossen und im Februar 1986 offiziell unterzeichnet wurde, ist wohl ein Meilenstein in der Vertiefung der Europäischen Gemeinschaft. Mit der ihm angeborenen Energie verfolgte Jacques Delors deren Umsetzung in über 300 Richtlinien. Dieser Vertrag war ohne Zweifel ein wesentlicher Schritt zur Überwindung der Stagnation des Integrationsprojektes und brachte neuen Auftrieb für Wirtschaft und Industrie.

Am Rande sei nur erwähnt dass dieses Ergebnis damals nicht so positiv beurteilt wurde. Das Europäische Parlament verhielt sich eher zurückhaltend, und sogar Delors war etwas verschnupft, weil er glaubte, wir hätten zu viele Kompromisse geschlossen. Der Generalsekretär der Kommission Emile Noël hat mir später bei seinem Abschiedsempfang vertraulich gesagt, er hätte nur einmal seinem Präsidenten die Gefolgschaft verweigert, und zwar als Delors ihn gebeten habe, ein negatives Kommuniqué zum Abschluss der Verhandlungen vorzubereiten. Diese Einstellung des Präsidenten hat sich allerdings schnell geändert, und ich bin froh, dass Delors in seinen Memoiren von der Einheitlichen Europäischen Akte sogar als „,mon traité favori“, also seinen Lieblingsvertrag, spricht, was er für spätere Verträge nicht sagen wolle.

\section{Meilenstein Währungsunion}

Die wirtschaftliche Integration wurde mit der weitgehenden Realisierung der Wirtschafts- und vor allem der Währungsunion zum 1. Januar 1999 erheblich vertieft und hat der Union psychologisch eine Ausstrahlung gegeben, die ihr Image weltweit gefestigt hat. Nicht zu Unrecht hat schon Walter Hallstein 1969 in seinem Werk „Die Europäische Gemeinschaft" die Währungspolitik als den Prüfstein für die Gemeinschaft bezeichnet. Nachdem die mitgliedstaatlichen Volkswirtschaften durch die anhaltenden, in fast regelmäßigen Abständen erfolgenden Währungsturbulenzen zwischen 1958 und 1969 erschüttert wurden, wurde auf dem Haager Gipfel 1969 beschlossen, dass der Prozess der Integration zu einer Gemeinschaft der Stabilität und des Wachstums führen müsse. Ein Stufenplan für die Einrichtung einer Wirtschafts- und Währungsunion sollte ausgearbeitet werden. Mein Amtsvorgänger Pierre Werner wurde hiermit beauftragt. Dieser legte schon am 8. Oktober 1970 seinen Schlussbericht vor. „Es ist das bedeutendste Dokument der europäischen Integration“ sagte Walter Hallstein. Und in der Tat wird im Werner-Plan resümiert: „Die WWU erscheint als ein Ferment für die Entwicklung der Politischen Union, ohne die sie auf die Dauer nicht bestehen kann.“

Welche Weitsicht und welcher Realitätssinn 1970! Das Ziel ist eine Union, so Werner, die ,,im Innern die vollständige und irreversible Konvertibilität der Währungen, die Beseitigung der Bandbreiten der Wechselkurse, die unwiderrufliche Festsetzung der Paritätsverhältnisse und die völlige Liberalisierung des Kapitalverkehrs bewirkt. Politische Gründe,“ 
so heißt es weiter, „sprechen für eine einheitliche Währung.“ Weiter schreibt Werner: „Die Union muss zugleich eine Wirtschaftsunion sein. Sie muss daher mit parallelen Fortschritten in der Konvergenz und später in der Vereinheitlichung der Wirtschaftspolitik verbunden sein." Das heißt also, dass Parallelität bestehen müsse zwischen der Schaffung dieser Kompetenzen und dem dafür notwendigen Aufbau von Gemeinschaftsinstitutionen. Als Institutionen schlug Werner „ein wirtschaftliches Entscheidungsgremium für die Fiskal- und Konjunkturpolitik und ein gemeinschaftliches Zentralbanksystem, das von Weisungen unabhängig ist" vor.

Leider kam es damals - zu Beginn der 1970er Jahre und zur Zeit der Sechsergemeinschaft - nicht zur Umsetzung des, Werner-Plans`. Die Ironie des Schicksals wollte es, dass erneute Währungsturbulenzen ausgelöst wurden durch die Entscheidung des amerikanischen Präsidenten Nixon vom 15. August 1971, die Leitwährung, den US-Dollar, für nicht mehr konvertibel zu erklären. Kaum ein anderes währungspolitisches Ereignis hat die freie Welt ähnlich erschüttert. Es zeigte sich, dass auch das reiche, mächtige Amerika seine Wirtschaft nicht ungestraft überlasten kann. Eine Lektion, die man heute noch beherzigen sollte.

Dann wurde es bis 1988 in Hannover still um die Wirtschafts- und Währungsunion. Damals wurde ein Ausschuss unter der Leitung von Jacques Delors eingesetzt, um sie neu zu beleben. Ich weiß nicht, welches Meister- oder sogar Husarenstück damals Helmut Kohl vollbrachte, um Margaret Thatcher zu bewegen, einem solchen Schritt zuzustimmen. Delors setzte den Werner-Plan in einen Stufenplan um, der in der Regierungskonferenz unter Luxemburger Präsidentschaft angenommen und zu einem wesentlichen Pfeiler des Maastrichter Vertrags wurde.

Dann wurde es erneut still um die Wirtschafts- und Währungsunion, doch als meine Kommission im Mai 1995 das Grünbuch zum Übergang zur Einheitswährung veröffentlichte entstand eine große Aufregung unter den Zentralbankern. Ich erinnere mich noch sehr gut daran, dass damals Bundesbankpräsident Hans Tietmeyer seine Ferien am Tegernsee unterbrach und mich in Brüssel aufsuchte, um eine gemeinsame Erklärung zu verfassen, die dann als Vorwort zum Grünbuch erschien.

Die Einführung der dritten Stufe der Wirtschafts- und Währungsunion gestaltete sich zusehends zu einer gewaltigen Kraftanstrengung. Nicht nur, dass jeder Schritt von uns der Wachsamkeit der nationalen Währungshüter unterzogen wurde, auch herrschte zu Beginn in internationalen Finanzkreisen eher Skepsis und Misstrauen. Ironische Kommentare seitens des damaligen amerikanischen Finanzstaatssekretärs Lawrence Summers erntete ich 1995, als ich in Davos die Ansichten der Kommission zur Wirtschafts- und Währungsunion vortrug. Nur wenige glaubten an die Realisierbarkeit eines solchen Projektes. In wie vielen Talkshows im deutschen Fernsehen wurden Wirtschaftsprofessoren nicht müde, die illusorischen Bestrebungen der Kommission zu brandmarken. All dies schüchterte mich nicht ein. Ein Weg war aufgezeichnet, und ich beschritt ihn mit Mut und Ausdauer. Langsam wendete sich das Blatt. So kam es, dass US-Präsident Bill Clinton mich 1997 ohne vorherige Absprache anlässlich eines Treffens der G7 aufforderte, einmal in dieser Runde zu erklären, was es eigentlich mit diesem Projekt einer einheitlichen europäischen Währung auf sich hätte.

\section{Letzte Hürde: Der Stabilitätspakt}

Die letzte Hürde, die es zu nehmen galt, war der von deutscher Seite initiierte Stabilitätspakt. Dies war höchst problematisch. Zuerst stieß er in der Kommission wie auch bei mehreren Mitgliedstaaten auf Ablehnung. Ich war mir allerdings bewusst, dass es ohne eine Einigung über den Stabilitätspakt nicht zur Währungsunion kommen würde. Ich versuchte deshalb, den Pakt aus der intergouvernementalen Sicht, in der er konzipiert wurde, in einen 
Gemeinschaftsrahmen zu setzen. Schließlich wurde auf dem Gipfel in Dublin 1996, als die Wellen hochschlugen und der Pakt auf des Messers Schneide stand, ein Kompromiss zwischen Frankreich und Deutschland erreicht. Hier spielte zweifellos Jean-Claude Juncker die Rolle des geschickten Maklers.

Erneut erleben wir die Ironie des Schicksals: Derjenige, der den Stabilitätspakt als conditio sine qua non zum Einstieg in die Währungsunion machte, hielt als Erster Bestimmungen nicht ein und verletzte dessen Geist. Derjenige, der als Hebamme dieses Paktes fungierte, war derselbe, der ihm sieben Jahre später (2005) eine flexiblere Form gab, ohne jedoch an seinen Fundamenten zu rütteln. Dies war ein gewagter Seiltanz ohne Auffangnetz auf höchster Ebene. Nicht zu Unrecht hat Juncker 2004 die Auszeichnung ,Das goldene Schlitzohr erhalten!

\section{Die politische Logik der Wirtschafts- und Währungsunion}

Wenn ich die Währungsunion eingehender behandle, dann weil ich der festen Überzeugung bin, dass diese Union mit der einheitlichen Währung, dem Euro, der einzige feste föderale Faktor ist, der die EU in diesen Krisenzeiten zusammenhält. Ich bin aber kein Monetarist, der wie der frühere französische Finanzminister Jacques Rueff zu sagen pflegt: „L'Europe se fera par la monnaie ou ne se fera pas“ (Europa wird durch die Währung geboren oder es wird kein Europa geben).

Gewiss entspricht die Währungsunion zuerst einer wirtschaftlichen Logik. Ich kann mir keinen integrierten Binnenmarkt vorstellen, der mit damals 14 verschiedenen Währungen zufriedenstellend funktionieren kann. Auch wird der Euro uns helfen, das gewaltige Potenzial dieses großen Binnenmarktes mit über 450 Millionen Verbrauchern voll auszuschöpfen. Auch haben wir mit dem Euro eine makroökonomische Konvergenz in der Eurozone erreicht, die ihresgleichen sucht. Wo stünde Europa heute nach den asiatischen, russischen und anderen Währungskrisen der letzten Jahre? Die Währungsschwankungen und -turbulenzen zwischen den Teilnehmerländern sind endgültig verschwunden. Wechselkursrisiken und Wechselgebühren sind weggefallen. Wir haben auf diesem Gebiet eine Kultur der Stabilität in den letzten Jahren erreicht, die wir seit dem Ende des Zweiten Weltkrieges nicht mehr kannten.

Aber hinter dem Euro steht auch und vor allem eine politische Logik. Der Euro wird zum bisher greifbarsten Zeichen der europäischen Einheit. Nichts ist so konkret wie die Währung. Nichts ist im Alltag der Europäer so sichtbar wie der Euro. Des Weiteren darf man nicht übersehen, dass die Währung ein verbindender Faktor ist und Ausdruck der Legitimation einer Nation, einer Gemeinschaft oder eines Staatenverbundes. Wir brauchten 40 Jahre zur Schaffung des Euro, die Amerikaner bekamen den Dollar erst nach über 60 Jahren. Der Euro macht die EU in der Weltwirtschaft schlagartig sichtbarer. Er hat, nach der anfänglichen Zitterpartie, seine Bewährungsprobe bestanden. Schon bei der Finanzkrise in Asien hat er als Schutzschild gedient. Dank der Währungsunion wurde Europa davon verhältnismäßig wenig berührt. Europa als neue Währungsgroßmacht muss sich nun die Frage nach der weltwirtschaftlichen Verantwortung stellen.

Zudem müssen wir uns heute mehr denn je die Frage stellen, ob wir das gesamte Potenzial der Wirtschafts- und Währungsunion erschöpft haben. Wie steht es mit der Umsetzung der Beschlüsse des Wiener Gipfels des Jahres 1998, nach denen in Währungsfragen die EU mit einer Stimme sprechen sollte? Zwar haben wir seit zwei Jahren einen Präsidenten der Eurogruppe, aber wie steht es mit dem Pendant zur Europäischen Zentralbank, nämlich der gemeinsamen Wirtschaftspolitik, die bereits im Werner-Bericht angemahnt wurde. Aus meiner eigenen Erfahrung als langjähriges Mitglied der belgisch-luxemburgischen Währungsu- 
nion, die seit 1921 funktionierte, weiß ich, dass eine solche Union nur Bestand haben kann, wenn es eine Konvergenz der Haushalts- und Wirtschaftspolitiken gibt. Es gab immer Schwierigkeiten in dieser Union, verbunden mit Währungsturbulenzen und -anpassungen, wenn es zu einem Auseinanderdriften dieser Politiken kam. Hier liegt also noch ein großes Betätigungsfeld vor uns.

\section{Die Europäische Gemeinschaft nach dem Fall der Mauer}

Man kann keine Bilanz der vergangenen 25 Jahre ziehen wenn man nicht die historischen Ereignisse der Jahre 1989 bis 1991 beleuchtet, welche das Ende des Kalten Krieges besiegelt, die Wiedervereinigung Deutschlands herbeigeführt und die geografische Erweiterung um die neuentstandenen Demokratien Mittel- und Osteuropas ermöglicht haben.

Vom europapolitischen Standpunkt aus gesehen verliefen diese Prozesse nicht ohne Schwierigkeiten und Befürchtungen, psychologisch allerdings haben sie zuerst in der europäischen Öffentlichkeit und dann weltweit zu einer gewaltigen Aufbruchstimmung geführt, die heute kaum mehr nachzuvollziehen ist. Ich kann mich noch allzu gut erinnern, dass in einer Konferenz ein bekannter und anerkannter Finanzguru von einem anbrechenden goldenen Zeitalter des Perikles sprach!

Die Wiedervereinigung Deutschlands hat zu Beginn zu gewissen Unruhen geführt. Die politische Klasse wurde durcheinandergewirbelt. Ich war damals Präsident der EVP und versuchte etwas Ordnung und Disziplin in die christdemokratischen Reihen zu bringen. So gelang es mir, ein eintägiges informelles Treffen aller christdemokratischen Regierungschefs in Pisa am Rande eines Kongresses der EVP-Jugend zu organisieren. Helmut Kohl überzeugte die skeptischen Kollegen, sowohl Giulio Andreotti als auch Ruud Lubbers und andere Parteiführer von der Bedeutung der Wiedervereinigung und seine von ihm gewollte Einbindung in die Europäische Union. Nicht wenig verdutzt war die Presse, als Gastgeber Andreotti - der noch kurz vorher sagte, er wolle lieber zwei deutsche Staaten als nur einen - die einmütige Zustimmung der Christdemokraten zur Wiedervereinigung Deutschlands bekundete!

Mit großem Geschick und dem notwendigen Einfühlungsvermögen verstand es Helmut Kohl, seine Partei (die EVP) für seine Pläne zu gewinnen und alle Befürchtungen eines hegemonialen Deutschlands durch eine starke Einbindung in die Europäische Gemeinschaft zu entkräften. Dies mag auch ausschlaggebend gewesen sein für das Drängen zur Einberufung der Regierungskonferenz zuerst zur Währungsunion und später zur politischen Union, die zum Maastrichter Vertrag führte.

\section{Die Erweiterung als Vereinigung des Kontinents in Frieden und Freiheit}

Als problematischer erwies sich die Heranführung der beitrittswilligen neuen Demokratien in Mittel- und Osteuropa. Mitterrand schlug zuerst im Dezember 1989 die Schaffung einer Konföderation vor, deren erste Sitzung in Prag im Juni 1991 ein Misserfolg war. Delors selbst war eher zurückhaltend und hatte mit Blick auf die notwendige Handlungsfähigkeit der Gemeinschaft, unterstützt von einzelnen Staaten, im Juni 1992 auf dem Gipfel in Lissabon eine vertiefte Kooperation mit den Staaten Mittel- und Osteuropas vorgeschlagen, etwa nach dem Vorbild der EFTA. In seinen Memoiren zeigt sich Delors sehr enttäuscht darüber, dass seine Vorschläge mit einer Handbewegung weggefegt worden waren, ohne ihnen auch nur die geringste Aufmerksamkeit zu schenken. Im Wortlaut - denn dieses Statement ist wichtig für den weiteren Verlauf der Dinge: „Notre grande déception a été que le [...] Conseil Européen a balayé d'une main nos remarques sans leur prêter l'attention nécessaire. La Commission a été invitée, sans ménagement, à taire ses interrogations, voire ses inquiétudes.“ 
Die Kommission wurde also aufgefordert, ihre Fragen und Befürchtungen im Hinblick auf die Erweiterung unverzüglich einzustellen. Fünf Jahre später, im Jahre 1997, hat meine Kommission dann nach langen wissenschaftlichen Vorarbeiten in der sogenannten Agenda 2000 die Erweiterungsstrategie in einem Stufenplan dargelegt, zusammen mit der Reform der internen Gemeinschaftspolitiken, der Agrarpolitik, der Strukturfonds und der finanziellen Vorausschau bis 2007.

Wie gesagt - es war ein Stufenplan: Zuerst sollte mit denjenigen Kandidaten verhandelt werden, die am ehesten die vorgeschriebenen Kopenhagener Kriterien für einen Beitritt erfüllen konnten, in einer späteren Runde mit den drei oder fünf nächsten und so weiter. Dem Antrag der Türkei auf Eröffnung von Beitrittsverhandlungen wurde nicht stattgegeben, sondern es wurde eine Konferenz geschaffen, an der alle beitrittswilligen und beitrittsfähigen Kandidaten teilnehmen sollten und in der über Gemeinschaftsprobleme in regelmäßigen Abständen diskutiert werden sollte. Das betraf nicht nur die Türkei, sondern auch Norwegen, die Schweiz und andere Länder. Dieser Plan wurde nach langen Diskussionen auf dem Gipfel in Luxemburg im Dezember 1997 einstimmig angenommen, aber bereits im Dezember 1999 - also zwei Jahre später - in Helsinki über den Haufen geworfen. Der ,Big Bang “ wurde dekretiert, und der Türkei wurde der Kandidatenstatus zuerkannt, und dies nach einer weniger als zehn Minuten dauernden Diskussion. Ich habe bis jetzt, trotz meiner Nachforschungen, nicht herausgefunden, was die Staats- und Regierungschefs zwei Jahre später bewogen haben mag, ihre Haltung aufzugeben, denn im Rat waren dieselben Mitglieder. Mit zwei Ausnahmen: Bundeskanzler Kohl und der Kommissionspräsident waren ausgewechselt worden!

Noch heute bin ich sehr enttäuscht darüber, dass wir als Politiker gleich welcher Parteien es nicht verstanden haben, dieses einmalige historische Werk unseren Mitbürgern zu vermitteln. Waren wir uns alle bewusst, dass dies nicht nur eine geografische Erweiterung war, sondern dass wir - das heißt unsere Generation - die einmalige Chance haben, unseren Kontinent erstmals seit dem 14. Jahrhundert in Frieden und Freiheit zu vereinen?

Ich hatte in meinem Innersten geglaubt, dass wir in diesem Ereignis eine neue Motivation für Europa begründen könnten, besonders für die jüngere Generation, die nicht den ausgeprägten emotionsgeladenen Bezug zu Europa hat wie die ältere Generation, die Europa auf den Ruinen des Zweiten Weltkrieges aufgebaut hat. Dies ist grundsätzlich fehlgeschlagen, und anstatt eine neue Motivation zu schaffen hat sich das Projekt der Erweiterung gegen Europa gewandt und dabei Sorgen und Befürchtungen bei Bürgern, Gewerkschaften und Arbeitnehmern entfacht. Waren wir zu ehrgeizig? Haben wir den Bogen überspannt und versäumt, den Bürger mitzunehmen? Fragen über Fragen, die wir in der immer noch anhaltenden Reflexionsphase über die Bewältigung der jetzigen Verfassungskrise beantworten müssen.

\section{Das einzigartige Zusammenwirken des deutsch-französischen Tandems}

In den letzten Jahren hat Europa sich von Grund auf verändert. Ganz gewiss hat sich die Integration Europas als die richtige Strategie erwiesen. Auch Rückschläge und Krisen ändern nichts an der Tatsache, dass die Europäische Gemeinschaft seit nunmehr über 50 Jahren als Stabilitätsanker nach innen wie nach außen entscheidend zu Freiheit und Sicherheit, Wohlstand und sozialem Frieden beigetragen hat. Die über die Verträge von Maastricht und Amsterdam zur Europäischen Union weiterentwickelte Gemeinschaft gehört heute zu den politisch attraktivsten und erfolgreichsten Regionen der Welt.

Zuvor belegte ich diese These durch drei positive Entwicklungen in den letzten Jahren: Die Schaffung des Binnenmarktes, die Gestaltung der Wirtschafts- und Währungsunion mit 
der Einführung einer einheitlichen Währung, dem Euro, und die Wiedervereinigung Deutschlands und die Erweiterung um zehn neue Mitgliedstaaten 2004. Diese einmalige positive Entwicklung hätte meines Erachtens nicht stattfinden können, wenn es nicht ein $\mathrm{Zu}-$ sammenspiel und ein Zusammentreffen von verschiedenen Faktoren während dieser Zeitspanne gegeben hätte.

Ein entscheidender Faktor war wohl das einzigartige Zusammenwirken des deutsch-französischen Tandems unter Kohl und Mitterrand, ein regelrechter Motor für die Integration Europas. Ob der Finanzkompromiss in Fontainebleau 1984, die Vollendung des Binnenmarktes, die Gestaltung der Währungsunion oder der Erweiterungsprozess nach 1989: Keiner dieser wesentlichen Integrationsschritte hätte ohne ihre Initiative und Zutun verwirklicht werden können. Hinzu kam das harmonische und vertrauensvolle Zuarbeiten des Kommissionspräsidenten Jacques Delors, der über zehn Jahre mit Temperament die Geschicke der Gemeinschaft leitete.

Dies soll nicht heißen, dass es nicht gelegentlich zu Schwierigkeiten kam und dieses Dreierverhältnis nie abgekühlt wäre. Dies war besonders 1989/90 der Fall, als es um die Einheit Deutschlands ging. Mitterrand war wirklich verunsichert und hatte nicht mit der Entschlossenheit Kohls gerechnet, der die Weichenstellung für die deutsche Einheit als irreversiblen Prozess eingeleitet hatte. Wie sonst hätte man die Begegnung Mitterrands in Kiew mit Michail Gorbatschow verstanden, oder seine Aufwartung bei Erich Honecker anlässlich des 40. Jahrestages der DDR? Auch war die Begeisterung Mitterrands nicht groß, als Kohl die Initiative zu einer politischen Union ergriff. Erst nach der Volkskammerwahl in der DDR im März 1990 begriff Mitterrand die Notwendigkeit weiterer Fortschritte in der politischen Integration. Gemeinsam mit Kohl präsentierte er dann im Frühjahr 1990 die Forderung nach Einberufung einer weiteren Regierungskonferenz, die einen Vertrag über eine politische Union erarbeiten sollte.

Hinzu kam auch ein Faktor, den man nicht unterschätzen sollte: Die politische Langlebigkeit der damaligen Staats- und Regierungschefs. Es kam nicht oft zu einem Wechsel, und wenn, dann in einer gewissen Kontinuität - dies erleichterte natürlich die persönliche Zusammenarbeit in entscheidenden Momenten.

\section{Politisierung der Institutionen}

Ein anderer Faktor, der diese 25-jährige Entwicklung prägte, war die zunehmende Politisierung der Institutionen, die zu einer tieferen Demokratisierung führte.

\section{Die Aufwertung des Europäischen Parlaments}

Die Tatsache, dass das Europäische Parlament 1979 direkt gewählt wurde ohne dass man sich auf eine Erweiterung seiner Befugnisse einigen konnte hat sich im Nachhinein bitter gerächt. Das Frustrationsgefühl des direkt gewählten Parlaments war groß, und so hat es zurecht versucht, seine Befugnisse auszuweiten. Dadurch, dass in den verschiedenen Verträgen der Union insgesamt neue Aufgabenbereiche zugeteilt wurden, etwa im Bereich des Umweltschutzes, der Forschung und der Stärkung der Kohäsion durch die Einheitliche Europäische Akte sowie die Wirtschafts- und Währungsunion, Bildung, Kultur, Gesundheitswesen, Verbraucherschutz und Sozialpolitik durch den Maastrichter Vertrag wurde der Druck des Parlaments immer stärker. Die Mitwirkungsrechte wurden ausgeweitet, und in allen Fragen des Binnenmarktes, in denen der Ministerrat Mehrheitsentscheidungen trifft, wurde dem Parlament grundsätzlich das Mitentscheidungsrecht zugestanden. Das Europäische Parlament wurde somit institutionell weitgehend gleichrangig mit dem Ministerrat. 
Wenige nationale Parlamente haben diese institutionelle Revolution zur Kenntnis genommen, und als sie sich der Tragweite der neuen Bestimmungen bewusst wurden, hat sich eine neue Front des Argwohns gegenüber dem Europäischen Parlament gebildet. Der Ruf nach einer Neuaufteilung der Kompetenzen gemäß dem Subsidiaritätsprinzip wurde immer lauter.

Darüber hinaus versuchte das Parlament seine Muskeln gegenüber den anderen Institutionen spielen zu lassen, um seinen politischen Einfluss geltend zu machen. Die Kommission ist die einzige Institution, die gegenüber dem Parlament verantwortlich ist. Obschon Kommission und Parlament eigentlich objektive Verbündete gegenüber Ministerrat und Europäischem Rat sein müssten, wurde gelegentlich die Kommission nolens volens Opfer der parlamentarischen Einpeitscher. Dies habe ich in eigener Sache erfahren, als ich Kommissionspräsident war, und ich möchte sagen: in demokratischer Weise durchgestanden. Da ich noch keine Autobiografie geschrieben habe und mich persönlich noch nie öffentlich über die Demission meiner Kommission äußerte, erlaube ich mir an dieser Stelle, einige persönliche Anmerkungen hierzu zu machen.

\section{Die schwierige Suche nach einem Kommissionspräsidenten}

Ich habe bereits dargelegt, aus welcher Konstellation heraus ich 1994 zunächst kein Präsidentschaftsanwärter war. Es lagen damals unter anderem drei Kandidaturen vor, zwei von Beneluxministerpräsidenten, Jean-Luc Dehaene und Ruud Lubbers, sowie vom britischen Vizepräsidenten Sir Leon Britten. Der aussichtsreichste Kandidat war Dehaene. Als außenstehender Beneluxministerpräsident wurde ich von meiner Partei, der EVP, beauftragt, eine Einigung zwischen meinen Kollegen herbeizuführen. Dies sollte in einer vertraulichen $\mathrm{Zu}-$ sammenkunft bei einem Notar in der Nähe von Maastricht geschehen. An diesem Treffen nahm auch Wilfried Martens als Parteivorsitzender teil. Es kam nicht zu der gewünschten Einigung, lediglich in einer Verfahrensfrage, derzufolge derjenige Kandidat, der bei der anstehenden Abstimmung weniger Stimmen erhielt, sich zugunsten seines Kollegen zurückziehen solle.

Es kam dann zur Abstimmung auf dem Gipfel in Korfu. Dehaene erhielt sieben oder acht Stimmen, Lubbers drei oder vier, Britten eine. Aber es kam nicht zur Anwendung unseres vereinbarten Verfahrens, da weder Dehaene noch Lubbers anwesend waren und die Botschaft wahrscheinlich nicht an ihre Stellvertreter weitergeleitet wurde. So vertagte der von den Strapazen des Tages sichtlich gezeichnete Andreas Papandreou die Sitzung umgehend auf den nachfolgenden Morgen. Kaum in unser gemeinsames Hotel zurückgekehrt, wurden wir wieder in die Sitzung zurückbeordert, um eine Einigung herbeizuführen. Dies war erfolglos, und ich beobachtete, wie während der Nacht intensive diplomatische Bewegungen im Hotel stattfanden!

Tags darauf nach der Eröffnung der Sitzung fragte Papandreou, ob keiner der Kandidaten sich zugunsten von Dehaene zurückziehen möchte. Stille in der Runde, bis John Major das Wort ergriff um zu sagen, dass er keinesfalls mit einem Kandidaten Dehaene einverstanden sein könne, ohne allerdings Gründe für diese Erklärung, die einem Veto gleichkam, zu geben. So waren wir in einer Sackgasse gelandet. Papandreou machte einen letzten Versuch, in dem er den scheidenden Kommissionspräsidenten Delors fragte, ob dieser nicht bereit sei, sein Mandat um ein Jahr verlängert zu sehen, um in der Zwischenzeit eine Klärung herbeizuführen. Dieser erklärte damals sibyllinisch: „Ich war Präsident einer Kommission von zwölf Mitgliedstaaten, ich möchte nicht Präsident einer Kommission von 15 Mitgliedstaaten sein.“ Als ich später Präsident einer Kommission von 15 Mitgliedstaaten wurde, wurde mir bewusst, was Delors mit seiner Aussage bezweckte. 
Kohl, der auf Papandreou folgende Ratspräsident, ergriff darauf das Wort, um zu erklären, dass die angehende deutsche Präsidentschaft eine Lösung vor den Sommerferien finden werde. Er berief kurzerhand eine Sitzung des Europäischen Rates für den 15. Juli in Brüssel ein.

Sofort wurden Spekulationen laut über die offene Kandidatur. Namen von markanten Persönlichkeiten wurden ins Feld geführt: Peter Sutherland, Giuliano Amato, Poul Schlüter und andere. Schließlich rief mich dann am 7. Juli, während des G7-Gipfels in Neapel, Kohl zu Hause an, um mir klarzumachen, dass ich nach Rücksprache mit allen anderen Mitgliedern des Rates der einzige sei, der Zustimmung fände. Ich fühlte mich geehrt, sagte Kohl allerdings, dass ich nicht kandidiere, da ich eben erst nach gewonnener Wahl mein neues Kabinett gebildet hätte, das am 13. Juli vereidigt werden sollte. Kohl ließ nicht locker und gab mir zu verstehen, dass die Präsidentschaft der Kommission für ein kleines Land von übergeordneter Bedeutung sei. Er gab mir dann Bedenkzeit bis Sonntagsmorgen, den 8. Juli. Nach Rücksprache mit dem Großherzog und der Ordnung meiner etwaigen Nachfolge in der Person von Jean-Claude Juncker habe ich dann meine Zustimmung gegeben. Mein neues Kabinett wurde aber trotzdem am 13. Juli vereidigt, und am 15. Juli wurde ich im Europäischen Rat mit stehenden Ovationen bedacht.

\section{Die Rolle des Europäischen Parlaments bei der Kommissionsinvestitur}

Trotz der Annahme der Kandidatur ließ ich mich zunächst als Ministerpräsident vereidigen. Denn hier kam wieder das Europäische Parlament ins Spiel. Das Parlament sollte seine Zustimmung geben, dies geschah in der Vergangenheit mittels einer Konsultation des Parlamentspräsidiums, in dem die einzelnen Fraktionen vertreten sind. Zwar hatte Helmut Kohl am Rande des Europäischen Rates vom 15. Juli das Präsidium konsultiert. Dies beschloss allerdings, dass es zu einer öffentlichen Debatte im Parlament mit abschließender Abstimmung kommen solle. Dies war eine rein politische Entscheidung. Ich war selbstverständlich bereit, dem Wunsch des Parlaments nachzukommen, war mir aber des Risikos voll bewusst. Es war schließlich ein neu gewähltes Parlament, das natürlicherweise hier eine vorzügliche Möglichkeit sah, seine ,raison d'être ' und seine politische Rolle zum Ausdruck zu bringen. Ich habe auch deutlich gemacht, dass ich als guter Demokrat die Entscheidung des Parlaments annehmen würde. Darüber hinaus beschloss das Parlament, ein Hearing der zukünftigen Kommissionsmitglieder durchzuführen und nach dieser Anhörungsprozedur eine kollektive Abstimmung über die gesamte Kommission vorzunehmen. Ich war der erste Präsidentschaftskandidat, der diese parlamentarischen Hürden nehmen musste. Aus Respekt gegenüber dem Willen des Parlaments, um nach außen nicht die Entscheidung vorweg nehmen zu wollen, blieb ich also Premierminister meines Landes.

Es ist überflüssig zu betonen, dass diese Prozedur sich alles andere als einfach gestaltete. Kontrovers waren die Debatten in einem neu gewählten Parlament, das sich seiner Vormachtstellung bewusst war und in denen offensichtlich nicht meine Person zur Diskussion stand - ich wurde eher zur Geisel des Europäischen Rates - sondern die Beziehungen des Parlaments zum Europäischen Rat und dem Ministerrat. Ich überstand die Abstimmung, allerdings nur mit einer Mehrheit von etwas mehr als 20 Stimmen. Die Vertreter der kleineren Staaten, egal welcher politischer Gruppierung sie angehörten, stimmten für mich. Nach heftigen Diskussionen stimmte auch die gesamte EVP-Fraktion mit Ausnahme eines Abgeordneten für mich. Auch die Kommissare hatten Mühe, sich bei den Hearings durchzusetzen. Es gab einige, die sich herber Kritik ausgesetzt sahen. In diesen Fällen musste ich selbst eingreifen, um etwaige Bedenken zu zerstreuen. Allerdings wurde dann die Kommission insgesamt mit einer Zweidrittel-Mehrheit im Amt bestätigt. 
$\mathrm{Zu}$ Recht weist Werner Weidenfeld in Ihrem Jahrbuch in seiner Bilanz der europäischen Integration für 2005 auf die neue Politisierung hin, und grundsätzlich bin ich mit Bezug auf meine eigene Erfahrung mit ihm einverstanden wenn er schreibt: „Für die politische Emanzipation des Parlaments gegenüber den nationalen Regierungen war dies vielleicht ein Befreiungsschlag. Und letztlich hat auch das Europäische Parlament kein Interesse an einer schwachen Kommission. Ihm ging es vielmehr darum, die Muskeln auch gegenüber den Mitgliedstaaten spielen zu lassen, die immer noch die Fäden bei der Zusammensetzung der Kommission in der Hand haben. Ohne eine konstruktive Zusammenarbeit mit der Kommission wird das Parlament aber nicht auskommen - denn beide sitzen im selben europapolitischen Boot." Ich würde hinzufügen: Aber nur die Kommission ist verantwortlich vor dem Parlament, nicht der Ministerrat.

\section{Der Rücktritt der Kommission Santer}

Am Ende des Mandats der Kommission, bereits im Vorfeld der 1999 anstehenden Direktwahl des Parlaments, kam es zur Anhäufung von sogenannten Korruptions- und Missmanagementsfällen, die zu einer Trübung der Beziehungen zwischen Kommission und Parlament führten. Bis auf zwei Fälle lag auch hier der Ursprung der Probleme Jahre, wenn nicht gar ein Jahrzehnt, vor dem Amtsantritt meiner Kommission. ${ }^{1}$ Ich persönlich habe nie die Verantwortung auf meine Vorgänger abgeschoben, weil ich der Meinung war, als Repräsentant einer Institution und in dieser Kontinuität die volle Verantwortung übernehmen zu müssen. In der Krisensituation, die sich zur offenen Auseinandersetzung mit dem Parlament entfachte, gebe ich zu, dass seitens der Kommission zwei strategische Fehler begangen wurden.

Erstens haben die Kommissare, die mit den zuständigen Parlamentsausschüssen in Kontakt waren (zum Beispiel mit dem Haushaltsausschuss), die Entschlossenheit des Parlaments unterschätzt. Als die Probleme auf den Tisch der Kommission kamen lag das Kind bereits im Brunnen und alle Schlichtungsanstrengungen, die ich als Präsident unternahm, kamen zu spät. Zu verhärtet waren damals schon die Fronten. Zweitens war es falsch, mich von meinen britischen Kollegen überreden zu lassen, nach dem Vorbild des britischen Unterhauses einen sogenannten „Ausschuss unabhängiger Sachverständiger“ einzusetzen und dessen Schlussfolgerungen zu akzeptieren.

Der Todesstoß gegen die Kommission kam letztendlich nicht vom Parlament, sondern von diesem Ausschuss, der zwar nach Prüfung der einzelnen Kritikpunkte bis auf eine Kommissarin alle Kommissare weißwusch, aber in seinen Schlussfolgerungen, die keinen Bezug auf die vorgebrachten Kritiken aufwiesen, die kollektive Verantwortung der Kommission anmahnte. Wir waren zu blauäugig anzunehmen, der „Ausschuss unabhängigerer Sachverständiger" wäre überparteilich, und wir übersahen, dass verschiedene Mitglieder aufgrund früherer Funktionen und parteipolitischen Ansichten noch ein paar Hühnchen mit der Kommission zu rupfen hatten. Im Nachhinein stellte sich heraus, dass diese Schlussfolgerungen auch nicht einstimmig abgefasst wurden. So demissionierte gleich anschließend ein Mitglied aus ,persönlichen' Gründen, ein anderes gab in Interviews zu verstehen, dass es die Schlussfolgerungen nicht so radikal verstanden hätte. Trotz alledem: Der Schaden war angerichtet, und Politiker und Journalisten stürzten sich auf das Dokument!

Die Kommission hatte zwei Möglichkeiten: sich dem Parlament zu stellen und womöglich in einem Misstrauensantrag zu unterliegen, oder zu demissionieren. Nach langen Dis-

1 Vgl. Waldemar Hummer/Walter Obwexer: Der ,geschlossene“ Rücktritt der Europäischen Kommission. Von der Nichtentlastung für die Haushaltsführung zur Neuernennung der Kommission, in: integration 2/1999, S. 77-94. 
kussionen wählten wir den zweiten Weg, die kollektive Demission, und zwar aus zwei Gründen. Erstens, wir waren am Ende unseres Mandats angelangt und hatten das von uns vorgesehene Legislativprogramm vollständig umgesetzt. Zweitens, und das war der springende Punkt, den nur wenige Beobachter zur Kenntnis genommen haben: Der Vertrag sieht vor, dass die Kommission nach dem notwendigerweise zu erfolgenden Rücktritt nach einem Misstrauensvotum im Parlament bis zur Einsetzung einer neuen Kommission nur noch geschäftsführend tätig sein kann. Bei einer kollektiven individuellen Demission dagegen - jedes Mitglied muss einzeln zurücktreten - kann der Ministerrat der Kommission das Vertrauen schenken, wodurch die Kommission befugt wird, ihre Aufgaben in voller Handlungsmacht auszuüben und gegebenenfalls neue Initiativen zu entwickeln. Nach Rücksprache mit der deutschen Ratspräsidentschaft wählten wir diesen Weg. Am Tage der Demission am 15. März 1999 hatte ich in Brüssel ein Gespräch mit Bundeskanzler Gerhard Schröder, der natürlich die ganze Angelegenheit bedauerte, mir aber und auch der Kommission das Vertrauen zusicherte, was dann auch der Ministerrat formell bestätigte. Das erlaubte uns, alle Legislativvorschläge der auf dem Berliner Gipfel beschlossenen Reformen der Agenda 2000 durchzuziehen und auch neue Initiativen zu ergreifen. So wurde etwa noch vor dem Abgang der Kommission die Reform des Wettbewerbsrechts in die Wege geleitet. Ich selbst wurde einen Tag später von meiner Partei zur Überraschung und zum Entsetzen vieler Parlamentarier zum Spitzenkandidaten bei den bevorstehenden Direktwahlen des Europäischen Parlaments bestimmt. Ich hatte einen entsprechenden Erfolg und erhielt mehr Präferenzstimmen als alle Spitzenkandidaten der konkurrierenden Parteien zusammen!

Ich habe eine direkte Schlussfolgerung aus dieser leidvollen Erfahrung gezogen, nämlich dass die Rolle und Funktion des Kommissionspräsidenten, besonders in einer erweiterten Union - ich war schließlich der erste Präsident einer Kommission mit 20 Kommissaren aus 15 Mitgliedstaaten - gestärkt werden muss. Ihm sollte ein weit größeres Mitspracherecht bei der Bestellung der Kommissare eingeräumt werden. Das Kollegium muss bei einer Erweiterung gestrafft werden. Doch vor allem muss dem Kommissionspräsidenten das Recht zugestanden werden, ein individuelles Mitglied unter gewissen Bedingungen zu entlassen und eine neue Kompetenzverteilung innerhalb der Kommission vorzunehmen. Ich habe heute die Genugtuung festzustellen, dass diese Auffassung weite Kreise gezogen hat und in den nachfolgenden Verträgen in Ansatzpunkten aufgenommen wurde.

\section{Europäische Wirtschaftsunion}

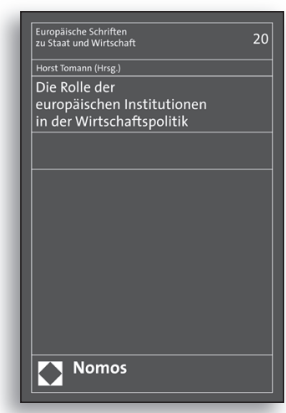

\section{Die Rolle der europäischen Institutionen in der Wirtschaftspolitik}

Herausgegeben von Prof. Dr. Horst Tomann

2006, 170 S., brosch., 34,- $€$, ISBN 3-8329-2165-6

Europäische Schriften zu Staat und Wirtschaft, Bd. 20

Die Autoren untersuchen offene Fragen der Kompetenzabgrenzung und Koordinierung in wichtigen Bereichen der Europapolitik. Binnenmarkt, Stabilitäts- und Beschäftigungspolitik und Strukturpolitik kommen auf den Prüfstand. 